\title{
NOTE ON COHOMOLOGY RING OF CERTAIN SPACES
}

\section{HIROSI TODA}

1. Statement of results. The purpose of this note is to survey the structure of the cohomology ring of a simply connected topological space $X$ having only nontrivial cohomology groups $H^{\text {in }}\left(X, Z_{p}\right) \approx Z_{p}$ for $i=0,1,2, \cdots, k$.

For such a space $X$, there exist always a cell complex $K=S^{n}$ $\cup e^{2 n} \cup \ldots \cup e^{k n}$ and a continuous mapping $f$ of $K$ into $X$ such that $f$ induces a ring isomorphism $f^{*}: H^{*}\left(X, Z_{p}\right) \approx H^{*}\left(K, Z_{p}\right)$. So we shall devote to consider the case $X=K$, and in particular $X=K=S^{n}$ $\cup e^{2 n} \cup e^{3 n}$.

We consider that all cells of the complex $K=S^{n} \cup e^{2 n} \cup e^{8 n}$ have orientations (generators)

$$
u \in H^{n}(K, Z), \quad v \in H^{2 n}(K, Z) \text { and } w \in H^{3 n}(K, Z) .
$$

Then the ring structure of $H^{*}(K, Z)$ is completely determined by two integers $a$ and $b$ such that

$$
u^{2}=a v \text { and } u v=b w .
$$

We shall say that an oriented complex $K=S^{n} \cup e^{2 n} \cup e^{3 n}$ has a type $(a, b)$ if the above relations hold.

Our main results are stated as follows.

Theorem 1. A complex $K=S^{n} \cup e^{2 n} \cup e^{3 n}$ of type $(a, b)$ exists if and only if one of the following conditions are satisfied.

(i) $n$ is odd and $a=0$.

(ii) $n=2$ or $n=4$.

(iii) $n$ is even, $n \neq 2,4,8, a \equiv 0(\bmod 2)$ and $a b \equiv 0(\bmod 3)$.

(iv) $n=8, a \equiv 0(\bmod 2)$ and $a b \equiv 0(\bmod 3)$.

(iv) $1 n=8, a \equiv 1(\bmod 2)$ and $a b \equiv 0(\bmod 24)$.

THEOREM 2. There exists no simply connected space $X$ having the truncated polynomial ring $Z_{p}[u] /\left(u^{k+1}\right)$ as its cohomology ring unless $\operatorname{dim} u=2,4$, where $p=2,3$ and $k \geqq 3$.

2. Lemmas. Let $\alpha \in \pi_{2 n-1}\left(S^{n}\right)$ be the homotopy class of the attaching map of $e^{2 n}$. Then it is well-known that the Hopf invariant $H(\alpha)$ of $\alpha$ is $\pm a$ (cf. [11]), and the following lemma is the same as Theorem 1.2 of [5].

Lemma 1. A complex $K=S^{n} \cup e^{2 n} \cup e^{3 n}$ of type $(a, b)$ exists if and

Received by the editors November 13,1961 . 
only if there exists an element $\alpha$ of $\pi_{2 n-1}\left(S^{n}\right)$ such that $H(\alpha)=a$ and $b\left[\alpha, \iota_{n}\right] \in \alpha \circ E^{n-1} \pi_{2 n-1}\left(S^{n}\right)$.

If there is such an element $\alpha$, then the element $k \alpha$ satisfies $H(k \alpha)$ $=k a$ and $h b\left[k \alpha, \iota_{n}\right] \in k \alpha \circ E^{n-1} \pi_{2 n-1}\left(S^{n}\right)$, for arbitrary integers $k$ and $h$. Thus,

CoRollary. If there exists a complex of type $(a, b)$, then there exist also complexes of types $(k a, h b)$ for arbitrary integers $k$ and $h$.

From the general expansion formula, for Whitehead product $[\alpha \circ \gamma, \beta]$, given by Barcus-Barratt [2], we have particularly

Lemma 2. $\left[\alpha, \iota_{n}\right]=\left[\iota_{n}, \iota_{n}\right] \circ E^{n-1} \alpha-H(\alpha)\left[\left[\iota_{n}, \iota_{n}\right], \iota_{n}\right]$ for $\alpha \in \pi_{2 n-1}\left(S^{n}\right)$.

Here, $H$ satisfies $H\left[\iota_{n}, \iota_{n}\right]=1+(-1)^{n}$, changing $H$ in sign if it is necessary.

LEMMA 3. There exists a complex of type $(0,1)$ for arbitrary $n$. If $n$ is even, then there exists complexes of types $(2,3)$ and $(6,1)$. If $n=2$ or 4 , then there exists a complex of type $(1,1)$.

In fact, the product $S^{n} \times S^{2 n}$ and complex (quaternion) projective 3-space are first and last examples. By Jacobi's identity for Whitehead product $[8], 3\left[\left[\iota_{n}, \iota_{n}\right], \iota_{n}\right]=0$. If $n$ is even, then $H\left(\left[\iota_{n}, \iota_{n}\right]\right)=2$. These relations prove the second assertion by setting $\alpha=\left[\iota_{n}, \iota_{n}\right]$ or $=3\left[\iota_{n}, \iota_{n}\right]$.

LEMmA 4. Let $n$ be even and $n \geqq 4$. There exists an element $\theta \in \pi_{3 n-3}\left(S^{n-1}\right)$ of order 3 such that $E \theta=\left[\left[\iota_{n}, \iota_{n}\right], \iota_{n}\right]$.

PROOF. In this case the mod 3 Hopf invariant: $\pi_{3 n}\left(S^{n+1}\right) \rightarrow Z_{3}$ is trivial $[6 ; 10]$. Then $\left[\left[\iota_{n}, \iota_{n}\right], \iota_{n}\right] \neq 0$ by Proposition 2.5 of $[13]$. Since $3\left[\left[\iota_{n}, \iota_{n}\right], \iota_{n}\right]=0$, then $\left[\left[\iota_{n}, \iota_{n}\right], \iota_{n}\right]$ is of order 3 . According to Serre [9], we have an isomorphism $\pi_{3 n-2}\left(S^{2 n-1}\right)+\pi_{3 n-3}\left(S^{n-1}\right) \rightarrow \pi_{3 n-2}\left(S^{n}\right)$ of the odd components. Then there are (uniquely) elements $\beta \in \pi_{3 n-2}\left(S^{2 n-1}\right)$ and $\theta \in \pi_{3 n-3}\left(S^{n-1}\right)$ such that

$$
\left[\left[\iota_{n}, \iota_{n}\right], \iota_{n}\right]=\left[\iota_{n}, \iota_{n}\right] \circ \beta+E \theta, 3 \beta=0 \text { and } 3 \theta=0 .
$$

According to G. W. Whitehead [16], we compute $\left(\iota^{\prime}+\iota^{\prime \prime}\right) \circ \gamma$ $-\iota^{\prime} \circ \gamma-\iota^{\prime \prime} \circ \gamma=\Gamma(\gamma)$, where $\iota^{\prime}$ and $\iota^{\prime \prime}$ denote the homotopy class of the canonical injections: $S^{n} \rightarrow S^{n} \bigvee S^{n}$ to the first and second factors. We have

$$
\Gamma\left(\left[\iota_{n}, \iota_{n}\right] \circ \beta\right)=\Gamma\left(\left[\iota_{n}, \iota_{n}\right]\right) \circ \beta=\left[\iota^{\prime}, \iota^{\prime \prime}\right] \circ 2 \beta
$$

since $\beta$ is a suspension element. Also we have 


$$
\begin{aligned}
\Gamma(E \theta)= & 0 \\
\Gamma\left(\left[\left[\iota_{n}, \iota_{n}\right], \iota_{n}\right]\right)= & {\left[\left[\iota^{\prime}, \iota^{\prime}\right], \iota^{\prime \prime}\right]+\left[\left[\iota^{\prime \prime}, \iota^{\prime}\right], \iota^{\prime}\right]+\left[\left[\iota^{\prime}, \iota^{\prime \prime}\right], \iota^{\prime}\right] } \\
& +\left[\left[\iota^{\prime \prime}, \iota^{\prime \prime}\right], \iota^{\prime}\right]+\left[\left[\iota^{\prime}, \iota^{\prime \prime}\right], \iota^{\prime \prime}\right]+\left[\left[\iota^{\prime \prime}, \iota^{\prime}\right], \iota^{\prime \prime}\right] \\
= & 0 \text { (Jacobi's identity). }
\end{aligned}
$$

Thus $\left[\iota^{\prime}, \iota^{\prime \prime}\right] \circ 2 \beta=0$. The correspondence: $\alpha \rightarrow\left[\iota^{\prime}, \iota^{\prime \prime}\right] \circ \alpha$ is an injection of $\pi_{3 n-2}\left(S^{2 n-1}\right)$ onto a direct factor of $\pi_{3 n-2}\left(S^{n} \vee S^{n}\right)$ [4], thus we have $2 \beta=0$. Since $3 \beta=0$, we have $\beta=0$ and $\left[\left[\iota_{n}, \iota_{n}\right], \iota_{n}\right]=E \theta$. Obviously $\theta$ is an element of order 3. q.e.d.

Lemma 5. Let $n$ be even, $n \geqq 6$ and let $m=n / 2$, then $E^{n-m-1}: \pi_{m+n-1}\left(S^{m}\right)$ $\rightarrow \pi_{2 n-2}\left(S^{n-1}\right)$ is a homomorphism onto the odd component of $\pi_{2 n-2}\left(S^{n-1}\right)$.

Proof. Consider the exact sequence

$$
\pi_{2 k+n-2}\left(S^{2 k-1}\right) \stackrel{E^{2}}{\rightarrow} \pi_{2 k+n}\left(S^{2 k+1}\right) \stackrel{j *}{\rightarrow} \pi_{2 k+n-2}\left(\Omega^{2}\left(S^{2 k+1}\right), S^{2 k-1}\right) .
$$

It is sufficient to prove that the above $E^{2}$ is a homomorphism onto of the $p$-primary components for $k \geqq[(m+1) / 2] \geqq 2$ and for odd prime p. By $[7 ; 12]$, the $p$-component of

$$
\pi_{2 k+n-2}\left(\Omega^{2}\left(S^{2 k+1}\right), S^{2 k-1}\right)= \begin{cases}0 & \text { for } 2 k+n-2<2 p k-2, \\ Z_{p} & \text { for } 2 k+n-2=2 p k-2 .\end{cases}
$$

The condition $k \geqq[(m+1) / 2]$ implies $2 k+n-2=2 k+2 m-2$ $<2 k+2(p-1) k-2=2 p k-2$ unless the case $p=3$ and $m=2 k$. In the case $p=3$ and $m=2 k$, the homomorphism $j_{*}$ is equivalent to the $\bmod 3$ Hopf invariant: $\pi_{6 k}\left(S^{2 k+1}\right) \rightarrow Z_{3}$. Since $k \geqq 2$, the mod 3 Hopf invariant is trivial and thus $j_{*}$ is a homomorphism which is trivial on 3-component. Therefore, in all cases, we have that $E^{2}$ is a homomorphism onto of the odd components, and thus the lemma has been proved. q.e.d.

COROLlaRy. Let $n$ be even and $n \geqq 6$. For an arbitrary element $\beta$ of $\pi_{2 n-2}\left(S^{n-1}\right)$, the composition $\beta \circ E^{n-1} \beta$ is contained in 2-component of $\pi_{3 n-3}\left(S^{n-1}\right)$.

Proof. By Lemma 5, $\beta=\beta_{1}+\beta_{2}$ and $\beta_{1}=E^{n-m-1} \beta^{\prime}$ for an element $\beta^{\prime}$ of odd components and an element $\beta_{2}$ of 2-component. Obviously, $\beta_{1} \circ E^{n-1} \beta_{2}=\beta_{2} \circ E^{n-1} \beta_{1}=0$ and $\beta_{2} \circ E^{n-1} \beta_{2}$ is contained in 2-component. By use of the reduced join $\beta^{\prime} \# \beta^{\prime}$, we have that $2\left(E \beta_{1} \circ E^{n} \beta_{1}\right)$ $=2\left(\beta^{\prime} \# \beta^{\prime}\right)=0$, i.e., the anti-commutativity of the composition [3] . By Serre's decomposition of $\pi_{3 n-2}\left(S^{n}\right)$, the suspension homomorphism $E: \pi_{3 n-3}\left(S^{n-1}\right) \rightarrow \pi_{3 n-2}\left(S^{n}\right)$ has the kernel of 2 -torsion. It follows that $\beta_{1} \circ E^{n-1} \beta_{1}$ is contained in 2-component. Consequently, we have that 
$\beta \circ E^{n-1} \beta=\beta_{1} \circ E^{n-1} \beta_{1}+\beta_{2} \circ E^{n-1} \beta_{2}$ is contained in 2-component. q.e.d.

Recall the following results (cf. [15]).

$$
\begin{aligned}
\pi_{14}\left(S^{7}\right) & \approx Z_{120}, \\
\pi_{15}\left(S^{8}\right) & =Z+E \pi_{14}\left(S^{7}\right) \approx Z+Z_{120}, \\
\pi_{n+7}\left(S^{n}\right) & =E^{n-8} \pi_{15}\left(S^{8}\right) \approx Z_{240} \quad \text { for } n \geqq 9 .
\end{aligned}
$$

The kernel of $E: \pi_{15}\left(S^{8}\right) \rightarrow \pi_{16}\left(S^{9}\right)$ is generated by the Whitehead product $\left[\iota_{8}, \iota_{8}\right]$.

Lemma 6. There are elements $\sigma \in \pi_{15}\left(S^{8}\right)$ and $\tau \in \pi_{14}\left(S^{7}\right)$ such that $H(\sigma)=1,\left[\iota_{8}, \iota_{8}\right]=2 \sigma-E \tau$ and the order of $\tau$ is 120. Thus $\sigma$ and $E \tau$ generate $\pi_{15}\left(S^{8}\right)$, and for $k \geqq 1, E^{k+1} \tau=2 E^{k} \sigma$.

In fact, there exists an element $\sigma$ of $\pi_{15}\left(S^{8}\right)$ such that $H(\sigma)=1$ and $E \sigma$ is of order 240. Then $\tau$ is determined by the formula $E^{2} \tau=2 E \sigma$.

Remark that we may handle the element $\sigma$ as the class of Hopf fibre map. In particular, the correspondence $(\alpha, \beta) \rightarrow \sigma \circ \alpha+E \beta$ gives an isomorphism:

$$
\pi_{i}\left(S^{15}\right)+\pi_{i-1}\left(S^{7}\right) \approx \pi_{i}\left(S^{8}\right) .
$$

Finally, we introduce the following result from Theorem 10.3 of [15].

LEMma 7. The order of the composition $\tau \circ E^{6} \sigma \in \pi_{21}\left(S^{7}\right)$ is 8 .

Since $2 \tau \circ E^{6} \sigma=\tau \circ E^{7} \tau$ belongs to 2-component, by the corollary of Lemma 5, we may replace in Lemma $7 \tau$ with $\sigma^{\prime}$ of [15].

\section{Proof of theorems.}

\section{Proof of Theorem 1.}

(i) The case $n$ is odd. By the anti-commutativity of the cup product, we have $2 u^{2}=2 a v=0$. Thus $a$ has to be zero. For arbitrary integer $b$, a complex $K$ of the type $(0, b)$ exists since Lemma 3 and the corollary of Lemma 1.

(ii) The case $n=2$ or $n=4$. The theorem is obvious by Lemma 3 and the corollary of Lemma 1 .

(iii) The case $n$ is even and $n \neq 2,4,8$. If a pair $(a, b)$ of integers satisfies $a \equiv 0(\bmod 2)$ and $a b \equiv 0(\bmod 3)$, then there exists a complex $K$ of type $(a, b)$ since Lemma 3 and the corollary of Lemma 1 .

Conversely assume that there exists a complex $K$ of type $(a, b)$ and apply Lemma 1 . Then there exists an element $\alpha \in \pi_{2 n-1}\left(S^{n}\right)$ such that $H(\alpha)=a$ and $b\left[\alpha, \iota_{n}\right] \in \alpha \circ E^{n-1} \pi_{2 n-1}\left(S^{n}\right)$. By the nonexistence of the element of Hopf invariant 1 in $\pi_{2 n-1}\left(S^{n}\right), n \neq 2,4,8[1]$, we have that $a \equiv 0(\bmod 2)$. Let $a=2 d . H(\alpha)=2 d=H\left(d\left[\iota_{n}, \iota_{n}\right]\right)$. Then it follows from the exactness of the sequence 


$$
\pi_{2 n-2}\left(S^{n-1}\right) \stackrel{E}{\rightarrow} \pi_{2 n-1}\left(S^{n}\right) \stackrel{H}{\rightarrow} Z
$$

that $\alpha=d\left[\iota_{n}, \iota_{n}\right]+E \beta$ for some $\beta \in \pi_{2 n-2}\left(S^{n-1}\right)$. Let $\gamma$ be an element of $\pi_{2 n-1}\left(S^{n}\right)$ such that $b\left[\alpha, \iota_{n}\right]=\alpha \circ E^{n-1} \gamma$. Then we have

$$
d b\left[\left[\iota_{n}, \iota_{n}\right], \iota_{n}\right]+b\left[E \beta, \iota_{n}\right]=d\left[\iota_{n}, \iota_{n}\right] \circ E^{n-1} \gamma+E\left(\beta \circ E^{n-2} \gamma\right)
$$

and thus

$$
\left[\iota_{n}, \iota_{n}\right] \circ\left(b E^{n} \beta-d E^{n-1} \gamma\right)=E\left(\beta \circ E^{n-2} \gamma-d b \theta\right)
$$

by use of Lemma 2 and Lemma 4. By use of Serre's decomposition of $\pi_{3 n-2}\left(S^{n}\right)$ in odd components and by use of the isomorphism $E: \pi_{3 n-3}\left(S^{2 n-2}\right) \approx \pi_{3 n-2}\left(S^{2 n-1}\right)$, we have that

$$
b E^{n-1} \beta-d E^{n-2} \gamma=\delta \text { and } \beta \circ E^{n-2} \gamma-d b \theta=\epsilon
$$

for some elements $\delta$ and $\epsilon$ of the 2-components of the corresponding homotopy groups. It is computed directly that

$$
d^{2} b \theta=b\left(\beta \circ E^{n-1} \beta\right)-\beta \circ \delta-d \epsilon .
$$

Obviously $\beta \circ \delta$ and $d \epsilon$ are contained in 2-component. By the corollary of Lemma $5, b\left(\beta \circ E^{n-1} \beta\right)$ and thus $d^{2} b \theta$ are contained in the 2component. Since $\theta$ has order 3 , then we have $a^{2} b=4 d^{2} b \equiv 0(\bmod 3)$ and thus $a b \equiv 0(\bmod 3)$. (iii).

(iv) 0 The case that $n=8$ and $a \equiv 0(\bmod 2)$ is proved similarly to

(iv) 1 The case $n=8$ and $a \equiv 1(\bmod 2)$. By use of the elements $\sigma$ and $\tau$ of Lemma 6 , any element $\alpha \in \pi_{15}\left(S^{8}\right)$ of $H(\alpha)=a$ is written by

$$
\alpha=a \sigma+t E r \quad \text { for some integer } t \text {. }
$$

By use of Lemma 2, Lemma 4 and Lemma 6, we have

$$
\begin{aligned}
{\left[\alpha, \iota_{8}\right] } & =a\left[\sigma, \iota_{8}\right]+t\left[E \tau, \iota_{8}\right] \\
& =\left[\iota_{8}, \iota_{8}\right] \circ\left(a E^{7} \sigma+t E^{8} \tau\right)-a E \theta \\
& =2(a+2 t)\left(\sigma \circ E^{7} \sigma\right)-(a+2 t) E\left(\tau \circ E^{6} \sigma\right)-a E \theta .
\end{aligned}
$$

Now assume that $a b \equiv 0(\bmod 24)$. By Lemma 4 and Lemma 7 , we have $a b \theta=0$ and $a b\left(\tau \circ E^{6} \sigma\right)=0$. Let $t=0$ and $\gamma=2 b \sigma$, then we have $\left[\alpha, \iota_{8}\right]=\alpha \circ E^{7} \gamma$. This shows the existence of a complex of type $(a, b)$.

Conversely, we assume the existence of a complex of type $(a, b)$ with $a \equiv 1(\bmod 2)$ and let $\alpha$ be the characteristic class of $e^{2 n}$ such that $H(\alpha)=a$. By Lemma 1 and Lemma 6, there exists an integer $s$ such that 


$$
b\left[\alpha, \iota_{8}\right]=\alpha \circ s E^{7} \sigma .
$$

It follows then from the above formula on $\left[\alpha, \iota_{8}\right]$ that

$$
(2 a b+4 b t-a s)\left(\sigma \circ E^{7} \sigma\right)=E\left((a b+2 b t-s t)\left(\tau \circ E^{6} \sigma\right)+a b \theta\right) .
$$

Concerning the direct sum decomposition, $\pi_{22}\left(S^{8}\right) \approx \pi_{22}\left(S^{15}\right)$ $+\pi_{21}\left(S^{7}\right)$, we have the equalities

$$
(2 a b+4 b t-a s) E^{7} \sigma=0 \text { and }(a b+2 b t-s t)\left(\tau \circ E^{6} \sigma\right)=-a b \theta \text {. }
$$

Since $E^{7} \sigma, \tau \circ E^{6} \sigma$ and $\theta$ have orders 240,8 and 3 respectively, we have that

$$
2 b(a+2 t) \equiv a s(\bmod 240), \quad b(a+2 t) \equiv s t(\bmod 8)
$$

and

$$
a b \equiv 0(\bmod 3) .
$$

Let $b=2^{x} b^{\prime}, s=2^{y} s^{\prime}$ and $t=2^{z} t^{\prime}$ for some integers $x, y, z \geqq 0$ and odd integers $b^{\prime}, s^{\prime}, t^{\prime}$. Then it follows that

$$
2^{x+1} \equiv 2^{y}\left(\bmod 2^{4}\right) \text { and } 2^{x} \equiv 2^{y+x}\left(\bmod 2^{3}\right) .
$$

If $x<3$, then we have $x+1=y$ and $x=y+z$. But this is impossible since $z \geqq 0$. Thus we have $x \geqq 3$ and $a b=8 a b^{\prime} \equiv 0(\bmod 8)$.

Consequently we have $a b \equiv 0(\bmod 24)$.

Proof of Theorem 2. There exist a cell complex $L=S^{n} \cup e^{2 n} \cup \ldots$ $\cup e^{k n}$ and a mapping $f: L \rightarrow X$ such that $f^{*}: H^{*}\left(X, Z_{p}\right) \rightarrow H^{*}\left(L, Z_{p}\right)$ is an isomorphism onto. (Cf. for example Lemma 4.13 of [14]). Then $H^{*}\left(L, Z_{p}\right)$ is a truncated polynomial ring. Let $K=S^{n} \cup e^{2 n} \cup e^{8 n}$ be the $3 n$-skeleton of $L$, then the type $(a, b)$ of $K$ satisfies $a \neq 0(\bmod p)$ and $b \neq 0(\bmod p)$. By Theorem 1 , this is possible only if $n=2$ or $n=4$ $(p=2$ or 3$)$.

\section{REFERENCES}

1. J. F. Adams, On the non-existence of elements of Hopf invariant one, Ann. of Math. (2) 72 (1960), 20-104.

2. W. D. Barcus and M. G. Barratt, On the homotopy classifications of the extensions of a fixed map, Trans. Amer. Math. Soc. 88 (1958), 57-74.

3. M. G. Barratt and P. J. Hilton, On join operations in homotopy groups, Proc. London Math. Soc. 3 (1953), 192-201.

4. A. L. Blakers and W. S. Massey, The homotopy groups of a triad. III, Ann. of Math. (2) 58 (1953), 409-417.

5. I. M. James, Note on cup products, Proc. Amer. Math. Soc. 8 (1957), 374-383.

6. A. L. Liulevicius, The factorization of cyclic reduced powers by secondary cohomology operations, Theses, The University of Chicago, 1960.

7. J. C. Moore, Some applications of homology theory to homotopy problems, Ann. of Math. (2) 58 (1953), 325-350. 
8. M. Nakaoka and H. Toda, On Jacobi identity for Whitehead products, J. Inst. Polytech. Osaka City Univ. Ser. A. 5 (1954), 1-13.

9. J.-P. Serre, Groupes d'homotopie et classes de groupes abeliens, Ann. of Math. (2) 58 (1953), 258-294.

10. N. Shimada, Triviality of the mod $p$ Hopf invariants, Proc. Japan Acad. 36 (1960), 68-69.

11. N. E. Steenrod, Cohomology invariants of mappings, Ann. of Math. (2) 50 (1949), 954-988.

12. H. Toda, On double suspension $E^{2}$, J. Inst. Polytech. Osaka City Univ. Ser A. 7 (1956), 103-146.

13. - p-primary components of homotopy groups of spheres. II, mod $p$ Hopf invariant, Memoirs Univ. Kyoto 31 (1958), 143-160.

14. - p-primary components of homotopy groups of spheres. IV, Toric constructions, Memoirs Univ. Kyoto 32 (1959), 297-332.

15. Composition methods in homotopy groups of spheres, Annals of Math. Studies 49, Princeton (to appear).

16. G. W. Whitehead, A generalization of the Hopf invariant, Ann. of Math. (2) 51 (1950), 192-237.

NORTHWESTERN UNIVERSITY AND

UNIVERSITY OF KYOTO

\section{A NOTE ON SUBGROUPS OF THE MODULAR GROUP1}

\section{MARVIN ISADORE KNOPP}

1. We will follow the notation of [4]. Let $\Gamma$ denote the $2 \times 2$ modular group, that is, the set of all $2 \times 2$ matrices with rational integral entries and determinant 1 . For each positive integer $m$ define $\Gamma(m)$, the principal congruence subgroup of level $m$, by

$$
\Gamma(m)=\left\{X=\left(\begin{array}{ll}
a & b \\
c & d
\end{array}\right) \in \Gamma \mid a \equiv d \equiv 1, b \equiv c \equiv 0(\bmod m)\right\} .
$$

Let

$$
T_{m}=\left(\begin{array}{cc}
1 & m \\
0 & 1
\end{array}\right)
$$

and let $\Delta(m)$ be the normal subgroup of $\Gamma$ generated by $T_{m}$. That is, $\Delta(m)$ is the smallest normal subgroup of $\Gamma$ containing $T_{m}$. Clearly, $\Delta(m) \subset \Gamma(m)$.

In [4] Reiner considers the following questions raised in [1]:

Received by the editors October 6, 1961.

${ }^{1}$ Research supported in part by National Science Foundation Grant No. G-14362 at the University of Wisconsin, Madison. 